\title{
Phages from Ganges River curtail in vitro biofilms and planktonic growth of drug resistant Klebsiella pneumoniae in a zebrafish infection model
}

\author{
Niranjana Sri Sundaramoorthy, Subramaniam Thothathri, Muthumeenakshi Bhaskaran, \\ ArunKumar GaneshPrasad ${ }^{*+}$ and Saisubramanian Nagarajan ${ }^{*+}$ (i)
}

\begin{abstract}
Bacteriophages are a promising alternative for curtailing infections caused by multi drug resistant (MDR) bacteria. The objective of the present study is to evaluate phage populations from water bodies to inhibit planktonic and biofilm mode of growth of drug resistant Klebsiella pneumoniae in vitro and curtail planktonic growth in vivo in a zebrafish model. Phage specific to K. pneumoniae (MTCC 432) was isolated from Ganges River (designated as KpG). One-step growth curve, in vitro time kill curve study and in vivo infection model were performed to evaluate the ability of phage to curtail planktonic growth. Crystal violet assay and colony biofilm assay were performed to determine the action of phages on biofilms. KpG phages had a greater burst size, better bactericidal potential and enhanced inhibitory effect against biofilms formed at liquid air and solid air interfaces. In vitro time kill assay showed a 3 log decline and a 6 log decline in K. pneumoniae colony counts, when phages were administered individually and in combination with streptomycin, respectively. In vivo injection of $\mathrm{KpG}$ phages revealed that it did not pose any toxicity to zebrafish as evidenced by liver/brain enzyme profiles and by histopathological analysis. The muscle tissue of zebrafish, infected with K. pneumoniae and treated with $\mathrm{KpG}$ phages alone and in combination with streptomycin showed a significant $77.7 \%$ and $97.2 \%$ decline in CFU/ml, respectively, relative to untreated control. Our study reveals that KpG phages has the potential to curtail plantonic and biofilm mode of growth in higher animal models.
\end{abstract}

Keywords: Bacteriophage, Klebsiella spp., Phage therapy, Ganges river

\section{Introduction}

Klebsiella pneumoniae is a Gram-negative nosocomial pathogen that can cause wide range of infections like pneumonia, upper respiratory tract infections, wound infections, diarrhea, urinary tract infection, bacteremia and septicemia (Jarvis et al. 1985). It is one of the leading causes of mortality and morbidity in bacterial

\footnotetext{
*Correspondence: arunkumar@scbt.sastra.edu; sai@scbt.sastra.edu ${ }^{\dagger}$ Arun Kumar Ganeshprasad and Saisubramanian Nagarajan Jointly communicated

Center for Research in Infectious Diseases (CRID), School of Chemical and Biotechnology, SASTRA Deemed University, Tamil Nadu, Thanjavur 613401, India
}

sepsis (Vading et al. 2018). Roughly, 54\% of K. pneumoniae strains that caused neonatal sepsis were observed to be multi drug resistant. As per WHO's antimicrobial resistant pathogens' list, carbapenem resistant Enterobacteriaceae falls under critical priority category (2017). Thus, there is an urgent need to devise new antimicrobials/resistance modulatory agents against Carbapenem resistant $K$. pneumoniae (CRKP). Due to evolutionary selection pressures, bacteria would invariably gain resistance to either existing or any novel antimicrobial agents/ even for resistance modulatory agents. In such a scenario, lytic phages would serve as a better choice, since; bacteriophages would serve as self-amplifying antimicrobial 
agents and development of resistance by bacteria against phages is a relatively manageable event with phage cocktail targeting different receptors than the development of antibiotic resistance (German and Misra 2001; Chan et al. 2016; Monferrer and Domingo-Calap 2019; Burmeister et al. 2020).

Phage therapy has been in vogue from 1930 until date, as evidenced by works from Eliava Institute of Bacteriophages Microbiology Virology (EIBMV) at Tbilisi, Georgia (Summers 2012). Commercial production of bacteriophages specific against Staphylococcus spp., Streptococcus spp., Pseudomonas spp., Proteus spp., Shigella spp. were done till mid 1950 's at EIBMV, HIEET, Poland (Sulakvelidze et al. 2001). Discovery of antibiotics, which exerted a broad spectrum of activity against bacteria and concern regarding use of bacterial viruses for therapy, led to decline in widespread use of phages for therapeutic purposes. Phages for human therapy to treat various ailments like skin infections, wound prophylaxis, burn wounds, respiratory infections and sepsis has been in practice for nearly 90 years (Abedon et al. 2011).

Phages specific for K. pneumoniae has been reported previously. Caecal filtrate of a healthy woman undergoing colonoscopy was shown to harbor lytic phage specific to $K$. pneumoniae sub spp. pneumoniae. The phage belonged to Siphoviridiae that harbored rosette like tail tip with depolymerase activity against capsular type K2 antigen and based on genome sequence analysis, it was classified under novel Kp36like virus (Hoyles et al. 2015). A lytic phage vB_KpnM- Teh.1 (Myoviridae), isolated from urban wastewater, was administered intraperitoneally to a BALB/c mice, immediately after an intranasal inoculation of K. pneumoniae, which resulted in a significant $7 \log$ reduction of bacterial bioburden (Sasani and Fereshteh Eftekhar 2020).

The objective of the present work is to study the efficiency of Klebsiella spp specific phages isolated from Ganges water, in curtailing drug resistant $K$. pneumoniae, both in planktonic and biofilm mode of growth, and also to test efficiency of lytic phages alone and in combination with antibiotic to mitigate in vivo infection using zebrafish as a model before proceeding to higher animal models. Towards this goal, in the present study, K. pneumoniae specific phage was isolated from Ganges water and its efficiency in curtailing planktonic and biofilm mode of growth in a drug resistant clinical isolate (MTCC 432) was evaluated both in vitro and in vivo in a zebrafish infection model.

\section{Materials and methods}

\section{Bacterial Strain and other materials}

K. pneumoniae sub spp. pneumoniae (MTCC 432), an isolate from human urinary tract, was obtained from
MTCC, IMTECH Chandigarh. The strain was grown in nutrient broth at $37{ }^{\circ} \mathrm{C}$ and preserved as glycerol stock at $-80{ }^{\circ} \mathrm{C}$. Antimicrobial profiling of the strain was performed by microbroth dilution method (Andrews and Andrews 2001). All media used in the study was procured from HiMedia Labs, India. Antibiotics, salts and other chemicals were procured from Sigma-Aldrich, USA or Sisco Reaearch Labs, India.

\section{Phage isolation and purification}

Bacteriophages specific against $K$. pneumoniae was isolated by inoculating an overnight culture of K. pneumoniae in the collected Ganges water, supplemented with 10X nutrient broth and incubated for $24 \mathrm{~h}$ at $37^{\circ} \mathrm{C}$. The enriched water was centrifuged and the supernatant, containing phages, was collected. The supernatant was filtered through $0.45 \mu \mathrm{m}$ filter (Whatman, GE Healthcare Life Sciences) and stored at $4{ }^{\circ} \mathrm{C}$ until titer determination. The bacteriophage titer was determined as described previously (Adams 1959). Briefly, the phage containing lysate was serially diluted in SM buffer (100 mM Sodium Chloride, $8 \mathrm{mM}$ Magnesium sulphate, $50 \mathrm{mM}$ Tris-cl $(\mathrm{pH} 7.5))$ and $100-300 \mu \mathrm{l}$ of each dilution was mixed with $100 \mu \mathrm{l}$ of early $\log$ phase of the host bacteria (MTCC 432). After incubation at $37^{\circ} \mathrm{C}$ for $20 \mathrm{~min}$, the phage-host suspension was mixed with $5 \mathrm{ml}$ of soft agar (0.7\% Luria Bertani Agar) and overlaid onto Nutrient agar plates, after incubation at $37{ }^{\circ} \mathrm{C}$ for $24 \mathrm{~h}, \mathrm{PFU} / \mathrm{ml}$ was determined. Plates showing 1-10 plaques was used to obtain homogenous plaque morphology by triple purification as mentioned earlier (Bonilla et al. 2016). Briefly, a single plaque was picked, resuspended in SM buffer, vortexed and centrifuged. The supernatant was serially diluted, incubated with host and plated as mentioned above for phage isolation. The process was repeated thrice ensuring that plaque morphology remained same during iterative process. The resulting phage lysate from triple purification was ultracentrifuged at $4{ }^{\circ} \mathrm{C}$ for $1 \mathrm{~h}$ at $30,000 \mathrm{rpm}$ to obtain concentrated, high-titer phage lysate. The phage lysate was preserved as phage banks at $4{ }^{\circ} \mathrm{C}$ for further experiments.

\section{Determination of host range}

In order to discern the host specificity of phage, spot test and liquid assay were performed. The phage was tested against different clinical isolates of $K$. pneumoniae (BC936, E474, U2016, BC1415, BC1994) and E. coli (U3790, U3176, U1007, U1024, U2354) obtained from Sundaram Medical Foundation, Chennai, India. In addition, the phage was also tested against reference strains of E. coli (MG1655), Acenitobacter baumannii, Enterobacter cloacae, Pseudomonas aeruginosa and Enterococcus faecalis. Spot assay was done as reported earlier (Zhao et al. 
2019). $300 \mu \mathrm{l}$ of fresh bacterial culture was added to soft agar, overlaid on nutrient agar plate and allowed to solidify. $5 \mu \mathrm{l}$ of the purified phage lysate $\left(10^{12} \mathrm{PFU} / \mathrm{ml}\right)$ were spotted on to the plate and incubated at $37^{\circ} \mathrm{C}$ overnight. For liquid culture based evaluation, $180 \mu \mathrm{l}$ of culture $(0.05 \mathrm{OD})$ was mixed with phage $\left(10^{8} \mathrm{PFU} / \mathrm{ml}\right)$ and incubated at $37^{\circ} \mathrm{C}$ (Xie et al. 2018). The bacterial growth was monitored by measuring absorbance at $595 \mathrm{~nm}$ at 0,30 , 60, 90, 120 and 240 min using Tecan Infinite ${ }^{\circledR}$ F50 Microplate reader (TECAN, Männedorf, Switzerland) and the growth curve was plotted. The experiment was carried out in triplicates.

\section{One Step growth curve}

A one-step growth curve of the phages was performed as reported earlier (Pajunen et al. 2000). The host bacteria was grown until early log phase $\left(0.4 \mathrm{OD}_{595}\right)$, centrifuged and resuspended in SM buffer. This was mixed with phages at a multiplicity of infection (MOI) of 1 and was allowed to adsorb for $5-10 \mathrm{~min}$ at $37{ }^{\circ} \mathrm{C}$. The mixture was then centrifuged, pellet was resuspended in nutrient media and incubated at $37^{\circ} \mathrm{C}$. Samples were withdrawn for every $5 \mathrm{~min}$ and were plated to determine phage titer. The experiment was performed in triplicates, and values depict the mean of three observations \pm standard deviation. Burst size was calculated as the ratio of average $\mathrm{PFU} / \mathrm{ml}$ in latent period to the average $\mathrm{PFU} / \mathrm{ml}$ of last three time points. The experiment was done in triplicates and the error bar represents standard error of the mean.

\section{$\mathrm{pH}$ Sensitivity of the isolated phage}

The $\mathrm{pH}$ stability of the phage and host specificity was evaluated as mentioned previously (Anand et al. 2020). The phages $\left(10^{7} \mathrm{PFU} / \mathrm{ml}\right)$ were incubated in buffers with varying $\mathrm{pH}$ (3.0, 5.0, 7.0, 9.0 and 11.0) for $1 \mathrm{~h}$. Post incubation, the phage titer was determined by agar overlay method and the average percentage of surviving phages was calculated from three independent experiments.

\section{Transmission electron microscopy (TEM)}

$5 \mu \mathrm{l}$ of high titer $\left(10^{10} \mathrm{PFU} / \mathrm{ml}\right)$ phage suspension was deposited on a carbon-coated copper grid and were allowed to adsorb for $1 \mathrm{~min}$. Phage particles were stained with $2 \%$ aqueous solution of uranyl acetate. Grids were examined with a FEI transmission electron microscope (Model JEM 2100F Jeol, Japan).

\section{Time kill assay}

Efficiency of the phages to curtail bacterial growth invitro over a time course of $24 \mathrm{~h}$ in broth culture was discerned using the time kill assay (Grillon et al. 2016). K. pneumoniae at a cell density of $10^{6} \mathrm{CFU} / \mathrm{ml}$ was inoculated into LB broth and Klebsiella spp. specific monophage $\left(10^{8} \mathrm{PFU} / \mathrm{ml}\right)$ was introduced into the culture. At different time points 0, 2, 4, 6 and $24 \mathrm{~h}$, samples were withdrawn and cells were plated on LB agar and plate counts were determined $24-48 \mathrm{~h}$ post incubation. Phage untreated culture was maintained as growth control. The experiment was performed in triplicates. Decline in bacterial counts relative untreated control would imply lytic potential of phages.

\section{Evaluation of phage-antibiotic combination}

The activity of phages in combination with various antibiotics-Streptomycin, meropenem, colistin, erythromycin, ciprofloxacin and tobramycin, were tested by microbroth dilution method. The antibiotics were serially diluted from $64 \mu \mathrm{g} / \mathrm{ml}$ to $1 \mu \mathrm{g} / \mathrm{ml}$ and $10^{8} \mathrm{PFU} / \mathrm{ml}$ of KpG phages were added onto the wells. $0.05 \mathrm{OD}\left(\sim 10^{6} \mathrm{CFU} /\right.$ $\mathrm{ml}$ ) of MTCC KP was inoculated and incubated overnight at $37^{\circ} \mathrm{C}$. After incubation, optical density at $595 \mathrm{~nm}$ was measured using Tecan Infinite ${ }^{\circledR}$ F50 Microplate reader. The ideal combination of phage and antibiotic, which effectively inhibited bacterial growth was selected and time kill study was performed as mentioned earlier. All experiments were performed in triplicates.

\section{Crystal violet assay}

Biofilms at liquid air interface is akin to biofilms on implantable medical devices (Christensen et al. 1985). Biofilms are formed with $K$. pneumoniae on 96-well micro titer plates with and without phages in Brain Heart Infusion (BHI) broth. Briefly, overnight culture was diluted in the ratio of 1:100 and $100 \mu \mathrm{l}$ of the diluted culture was added to the wells. $1 \mathrm{~h}$ post incubation at $37^{\circ} \mathrm{C}$, KpG phages at different dilutions were added. Appropriate untreated control and broth controls were maintained. $24 \mathrm{~h}$ post treatment, biofilms were washed with PBS to remove unbound cells, $125 \mu \mathrm{l}$ of $0.1 \%$ crystal violet was added and unbound crystal violet was washed off. The bound crystal violet, which is an indirect measure of EPS formed was extracted with $30 \%$ acetic acid and was quantified by measuring the absorbance at $595 \mathrm{~nm}$.

\section{Colony biofilm assay}

The protocol for forming colony biofilms was essentially as described previously (Merritt et al. 2005). Briefly, $13 \mathrm{~mm} 0.2 \mu \mathrm{m}$ membrane filters sterilized by UV radiation placed on sterile BHI agar and inoculated on the center with bacteria or bacteria along with phages. The ability of phages to decrease biofilm formation until $48 \mathrm{~h}$ was examined by visual observation.

\section{Fish toxicity studies}

Zebrafish (Danio rerio) measuring $\sim 4$ to $5 \mathrm{~cm}$ in length, weighing approx. $300 \mathrm{mg}$, was purchased from a local 
aquarium. The protocols adopted were approved by the Institutional Animal Ethics Committee (CPCSEA-510/ SASTRA/IAEC/RPP) of SASTRA Deemed to be University, India. Animal acclimatization was performed following previously established protocols (Phillips and Westerfield 2014). The study comprised of two groups: Phage untreated control fish and fish injected with phages. For toxicity evaluation, 6 fish were injected intramuscularly with $10^{10} \mathrm{PFU} / \mathrm{ml}$ of phages and mortality of the fish was monitored up to $48 \mathrm{~h}$. At the end of exposure ( $48 \mathrm{~h}$ ), fish was sacrificed (anesthetized by $150 \mathrm{mM}$ MS-222 and euthanized by decapitation), liver/ brain from two fish of the same group were pooled and homogenized in ice-cold buffer (Tris- $\mathrm{HCl}, 0.1 \mathrm{M}, \mathrm{pH}$ 7.4). The homogenate was centrifuged $(10,000 \mathrm{~g}, 10 \mathrm{~min}$, $4{ }^{\circ} \mathrm{C}$ ) to obtain supernatant, which was used for all analyses in duplicates. Brain acetyl-choline esterase and liver carboxyl esterase enzyme activities were determined as reported earlier (Christena et al. 2016). Acteylcholine iodide and $\alpha / \beta$ naphthyl acetate were used as substrates for determining brain and liver enzyme profiles, respectively. In order to evaluate if the phages induce inflammation or other immunological response, histopathological analysis was performed. Briefly, the muscle/liver tissue of the phage-injected fish were sectioned, subjected to Hematoxylin \& Eosin staining, histopathology analysis was performed using a bright field microscope (Nikon Eclipse $\mathrm{Ni}-\mathrm{U}$ ) and was compared with sham control.

\section{Zebrafish infection}

Intramuscular infection of zebrafish with $K$. pneumoniae strain was performed as described earlier (Neely et al. 2002). Briefly, $10 \mu \mathrm{l}\left(\sim 1 \times 10^{5} \mathrm{CFU} / \mathrm{ml}\right)$ of $K$. pneumoniae was injected intramuscularly to zebrafish (5/group) using a 3/10-cc U-100 insulin syringe with a 0.5-in.-long, 29 -gauge needle. $2 \mathrm{~h}$ post infection, phages $\left(10^{8} \mathrm{PFU} / \mathrm{ml}\right)$ were administered intramuscularly. Control fish group injected with $K$. pneumoniae was treated with equal volume of sterile buffer and both the groups were monitored. $48 \mathrm{~h}$ post treatment, fish from different groups were euthanized, injected muscle tissue was dissected, homogenized in sterile PBS. The homogenate was serially diluted and plated onto Luria Bertani agar plates and colony counts was determined after $24-48 \mathrm{~h}$ of incubation at $37^{\circ} \mathrm{C}$.

\section{Phage Identification by PCR}

Consensus primers for Podoviridae infecting Klebsiella species were designed using the PhiSiGns tool (http:// phisigns.sourceforge.net) (Dwivedi et al. 2012). To check the consistency of the primers, 60 different genomes of Podoviridae infecting Klebsiella spp were downloaded. The primers were checked for their ability to bind to the genomes manually. Two primer sets were designed and the sets 1 and 2 corresponds to T4 like DNA helicase/ primase and putative DNA primase of reference Klebsiella targeting Podoviridae phage genomes respectively. The primer sequences are as described in Table 1.50 ng of phage DNA isolated from cultured plaques was used as template for PCR. No-template controls were maintained. The conditions for PCR amplification are as follows: $95{ }^{\circ} \mathrm{C}-1 \mathrm{~min}, 30$ cycles of $95{ }^{\circ} \mathrm{C}-35 \mathrm{~s} ; 54{ }^{\circ} \mathrm{C}-15 \mathrm{~s}$; $72{ }^{\circ} \mathrm{C}-1 \mathrm{~min}$ and final extension at $72{ }^{\circ} \mathrm{C}-7 \mathrm{~min}$. The resultant PCR amplicons was sequenced using Sanger sequencer and its identity specifically to viral family Podoviridiae was further confirmed by BLAST analysis.

\section{Results}

Ganges river water was sampled at two different locations Haridwar, and Rishikesh. Attempts to isolate phages from these water sources by agar overlay method showed that only Ganges water sampled from Rishikesh displayed plaques of uniform morphology against MTCC 432 and was designated as KpG (Fig. 1). Although other sample from Haridwar displayed a lysis zone, it did not display distinct plaque morphology that could be further purified. Lack of phages from different water sources, for a ubiquitous strain like $K$. pneumonia, was rather uncommon. Hence, we checked whether the strain used in the present study produced capsule. Staining revealed

Table 1 Details of primers used in the study

\begin{tabular}{|c|c|c|c|c|c|}
\hline Sequence name & Sequence $\left(5^{\prime}-3^{\prime}\right)$ & $\begin{array}{l}\text { Expected } \\
\text { product size } \\
\text { (bp) }\end{array}$ & Reference Genome & $\begin{array}{l}\text { Location } \\
\text { of the expected } \\
\text { product in genome }\end{array}$ & Gene product \\
\hline CL1_FP & AAGGAGGGAATTACGGGATG & \multirow[t]{2}{*}{193} & \multirow{2}{*}{$\begin{array}{l}\text { KU183006.1 Klebsiella } \\
\text { phage vB_KpnP_- } \\
\text { IME205, complete } \\
\text { genome }\end{array}$} & \multirow[t]{2}{*}{$11,337-11,475$ bp } & \multirow[t]{2}{*}{ T4 like DNA primase/ helicae } \\
\hline CL1_RP & AC(A/G)ATGGAGCCATTCTGGTC & & & & \\
\hline CL2_FP & GGAATCATGCCTGAAATGGT & \multirow[t]{2}{*}{125} & \multirow{2}{*}{$\begin{array}{l}\text { NC_023567.2 Klebsiella } \\
\text { phage F19, com- } \\
\text { plete genome }\end{array}$} & \multirow[t]{2}{*}{$7593-7676 \mathrm{bp}$} & \multirow[t]{2}{*}{ Putative DNA primase } \\
\hline $\mathrm{CL} 2 \_\mathrm{RP}$ & ATTACCCACTTCGGTTGCTG & & & & \\
\hline
\end{tabular}




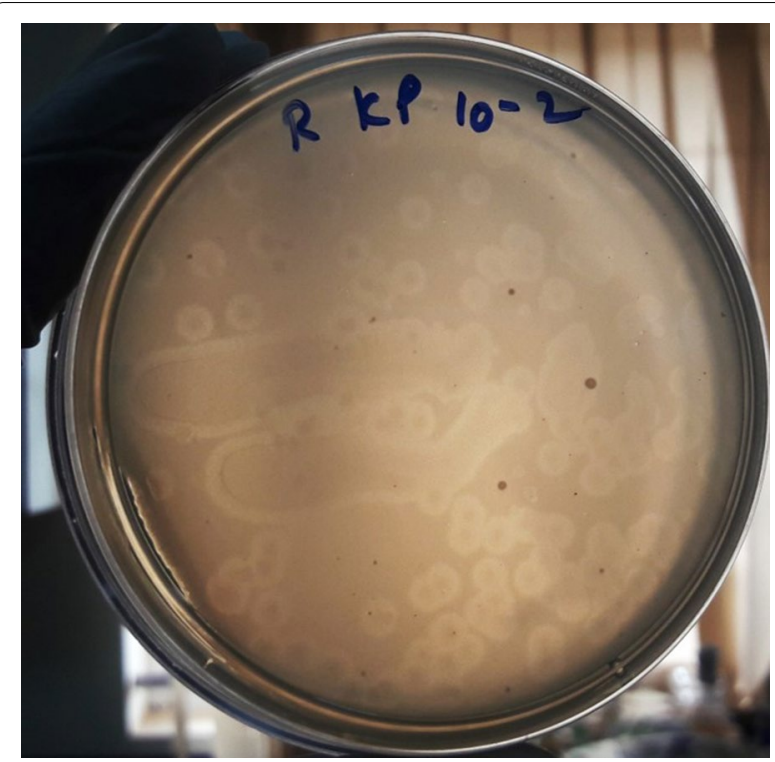

Fig. 1 Plaque morphology of the Klebsiella pneumoniae specific phages isolated from Ganges river (KpG) The Ganges water from Rishikesh was enriched with the host and plated by agar overlay method. KpG shows depolymerase activity, which is evident from the halo around the plaques

that MTCC strain indeed produced capsule (Additional file 1: Fig. S1). Morphology of plaques from Ganges water showed a halo around the clear zone, which might indicate depolymerase activity of KpG that could target the capsule of the strain (Fig. 1) (Domingo-Calap et al. 2020). The phage displayed stability at $\mathrm{pH}$ : 7.0, 9.0, 11.0 with a survival rate of $99 \%, 98 \%$ and $60 \%$ respectively (Fig. 2). Interestingly, we observed that plaques from $\mathrm{pH} 11.0$ group did not display even a faint halo around the lysis area, indicating the inability of these phages to produce depolymerase at $\mathrm{pH} 11.0$ (Additional file 1: Fig. S2). In order to check if KpG is specific to its capsulated $K$. pneumoniae host, spot test and liquid assays were performed against diverse bacterial strains viz., other capsulated strains of $K$. pneumoniae (BC936, E474, U2016) and uncapsulated strains of K. pneumoniae (BC1415 and BC1994), E. coli, A. baumannii, P. aeruginosa, E. faecalis, E. cloacae. We did not observe any lysis in these different bacteria except MTCC 432 strain (Additional file 1: Fig. S3), implying host tropism (specificity) of phages to a particular capsular variant of K. pneumoniae (MTCC 432).

KpG phage was purified by triple plaque purification method and its $\mathrm{PFU} / \mathrm{ml}$ were determined. Onestep growth curve of KpG phage revealed (Additional file 1: Fig. S4) a larger burst size of $224 \mathrm{PFU} /$ cell and a latent period of $20 \mathrm{~min}$. TEM images of the KpG suggested it belonged to Podoviridae (Fig. 3). We designed

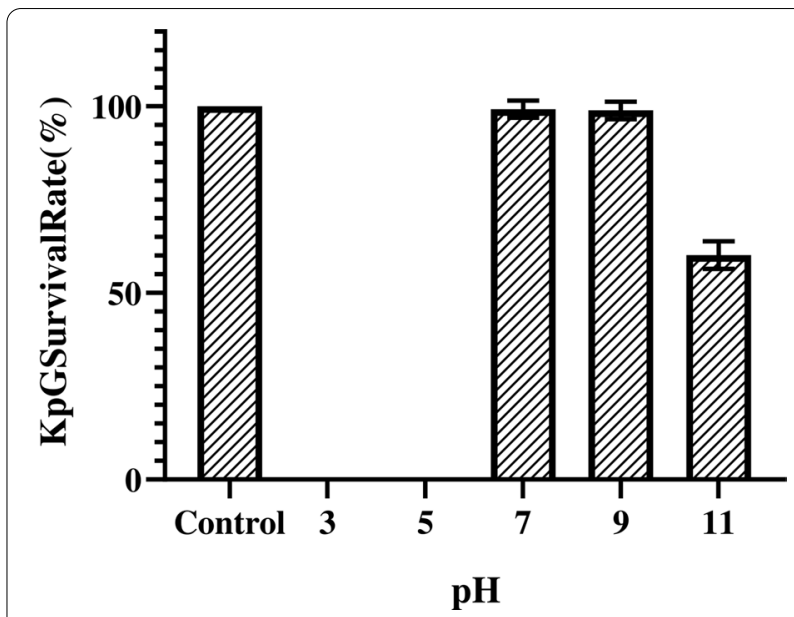

Fig.2 Stability of $\mathrm{KpG}$ at different $\mathrm{pH} .10^{7} \mathrm{PFU} / \mathrm{ml}$ of purified phage lysate was incubated in buffers of varying pH 3.0, 5.0, 7.0, 9.0 and 11.0 for $1 \mathrm{~h}$. Phage titer was estimated using agar overlay method and survival percentage was calculated using phage titers measured in SM buffer ( $\mathrm{pH}$ 6.8) as control. The experiment was performed in triplicates

two PCRs for identification of Podoviridae by analyzing the conserved regions of their genomes. We obtained $295 \mathrm{bp}$ and $97 \mathrm{bp}$ amplicons for CL1 and CL2 respectively (Additional file 1: Fig. S5). The PCR amplicons were sequenced by Sanger sequencing and have been submitted in Genbank (Accession numbers: CL1-MW026685

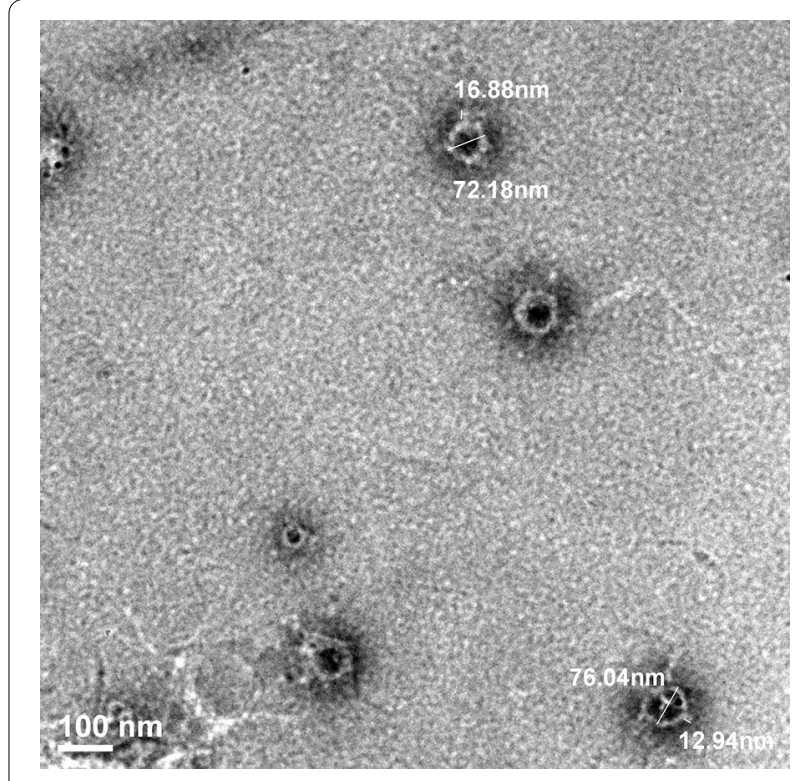

Fig. 3 TEM analysis showed that KpG phages belonged to the family Podoviridae. Triple purified Phage lysate of high titer was stained with $2 \%$ uranyl acetate and visualized under FEl transmission electron microscope (Model JEM 2100F Jeol, Japan). Image presented is representative of multiple images 
and CL2-MW026686). The sequences were further subjected to BLAST analysis against nucleotide database of NCBI and results from BLAST analysis revealed that CL1 amplicons exhibited a high degree of similarity ((97-98\% similarity with e score $1.55 \mathrm{e}^{-59}$ ) with T7-like phage primase/helicase protein Accession No: MN149903.1; Location: 18,733-18,871 and CL2 amplicons exhibited greater similarity $\left(96.7 \%\right.$ similarity with an e score of $\left.5.88 \mathrm{e}^{-29}\right)$ with putative DNA primase (Accession No: KP708986.1 Location: 7283 - 7366) with genome sequences of Klebsiella phages belonging to Podoviridae. Both Morphology by TEM and Sequence similarity with highly conserved region of Podoviridae showed that KpG isolate also belongs to family Podoviridae.

Time kill studies performed with KpG phages at an MOI of 1 , resulted in $0.5 \log$ decline initially but, significant regrowth was obtained at $24 \mathrm{~h}$ (data not shown). Hence, time kill curve was performed with a MOI of 100 , at which KpG phages caused an initial 3-4 log decline in bacterial cell counts by $4 \mathrm{~h}$ (Fig. 4). However by $24 \mathrm{~h}$, regrowth in bacterial cell counts were still observed, which might be due to evolution of phage resistant mutants, which was evident by allowing phages to interact with both phage exposed cells and unexposed cells. At different dilutions tested, phage unexposed cells of MTCC 432 strain was lysed by KpG phage whereas, when the same strain was exposed to KpG phages for $5 \mathrm{~h}$, the cells were resilient to lysis at all dilutions of KpG phage

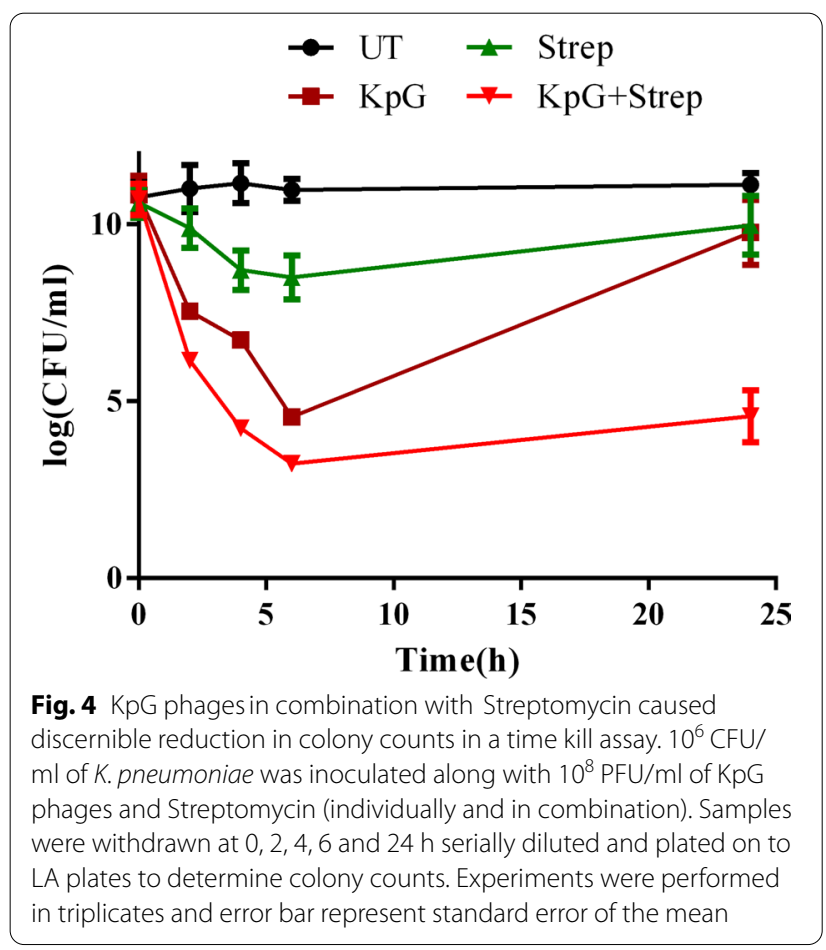

tested (Additional file 1: Fig S6), implying that exposure to the phage for $5 \mathrm{~h}(\sim 10$ generations) triggers evolution of phage resistant mutant. Capsular staining of strain by $5 \mathrm{~h}$ revealed that strain possessed capsule (data not shown) and inability of phages to lyse could not be attributed to differential expression of capsular polysaccharide. A similar phenomenon was reported earlier (Holst Sørensen et al. 2012).

In order to circumvent the evolution of phage resistant mutant, we tested the phages in combination with different antibiotics (Streptomycin, meropenem, colistin, erythromycin, ciprofloxacin and tobramycin) against $K$. pneumoniae. We found that the combination of streptomycin and phages were effective in inhibiting the growth of $K$. pneumoniae, in addition, phages reduced the MIC of streptomycin by eightfold (from $64 \mu \mathrm{g} / \mathrm{ml}$ to $8 \mu \mathrm{g} / \mathrm{ml}$ ) implying synergy of KpG phages with streptomycin. We further evaluated the combination in vitro in a time kill assay. As expected, the combination of streptomycin and KpG caused a 7-log decline relative to the untreated control within $6 \mathrm{~h}$ and by $24 \mathrm{~h}$ despite a slight regrowth, a decline of $6 \log$ relative to the other groups was maintained (Fig. 4). Streptomycin treatment alone did not show significant reduction in colony counts at $24 \mathrm{~h}$.

Ability of phages to inhibit biofilms formed at liquid air interface was discerned by crystal violet assay, which showed that KpG phages caused a significant fivefold decrease in biofilm formation at $10^{-8}$ dilution relative to biofilm formed by untreated bacteria (Fig. 5a). K. pneumoniae can also cause wound infections in immune compromised persons, wherein it typically forms biofilms at solid air interface, in order to mimic biofilms at solid air interface, colony biofilms of $K$. pneumoniae were formed and ability of phages to inhibit colony biofilms were evaluated. The results revealed that treatment with KpG phages caused a substantial decline in colony biofilm formation at 24 and $48 \mathrm{~h}$ relative to the untreated control (Fig. 5b). Thus KpG phages displayed potent biofilm inhibitory activity against MTCC 432 strain of $K$. pneumoniae.

In order to discern whether biofilm inhibitory effect of phages could be attributed to lytic potential of phages, live dead imaging was performed on phage treated biofilms. K. pneumoniae were allowed to form biofilms on glass slides and were treated immediately with KpG phages and $24 \mathrm{~h}$ post treatment, the unbound cells were washed and slide was stained with live/dead imaging kit as per manufacturer's protocol and was imaged using Nikon Fluorescent microscope (Nikon Eclipse Ni-U, Nikon, Japan). Live/Dead imaging showed that treatment with KpG phages caused substantial proportion of dead cells as evidenced by yellow cells in merged image indicative of dead cells and only a small sub population were 
a

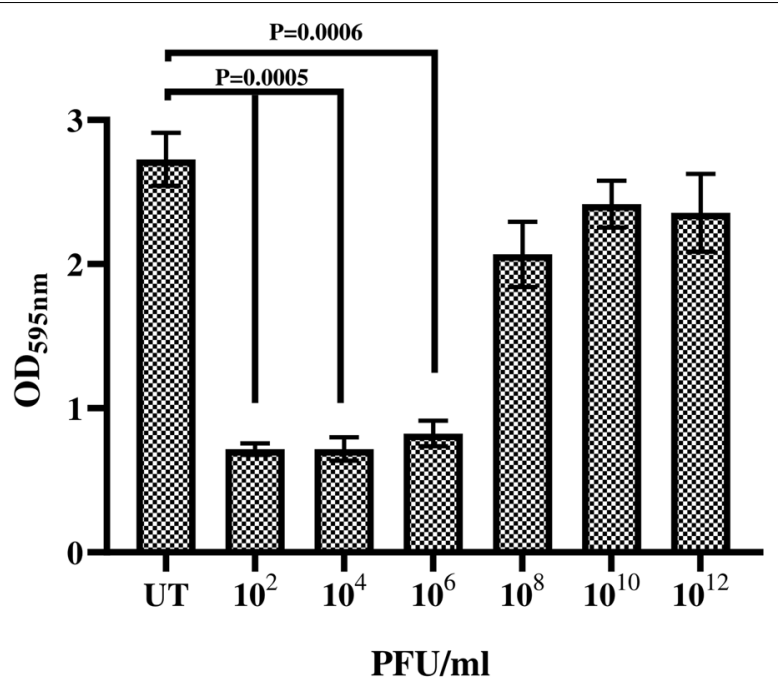

b

( h

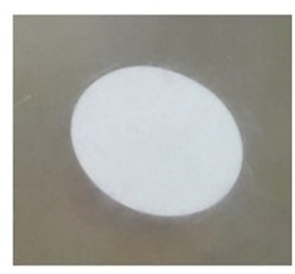

Untreated

KpG Treated

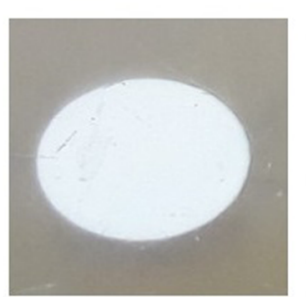

$24 \mathrm{~h}$
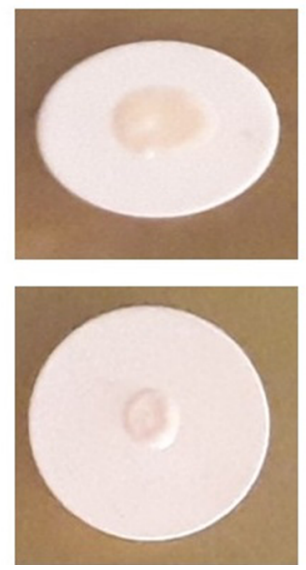

$48 \mathrm{~h}$
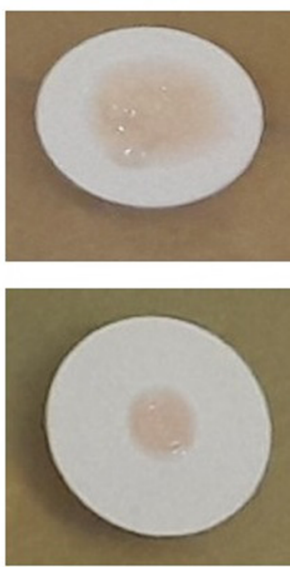

Fig. $5 \mathrm{KpG}$ phages efficiently inhibited biofilm formation of K. pneumoniae at liquid-air and at solid-air interface. a) Biofilms were formed in micro titer plates with or without KpG phages and washed with PBS. $24 \mathrm{~h}$ post treatment, Crystal violet was added and after 15 min, the unbound crystal violet was removed and the stain was extracted by acetic acid and the absorbance was measured at $595 \mathrm{~nm}, \mathrm{~b}$ ) Membrane filters were placed on $\mathrm{BHI}$ agar and inoculated with bacteria with or without KpG. The ability of phages to decrease biofilm formation can be examined visually till $48 \mathrm{~h}$. Images are representative of three independent experiments

alive indicating lytic potential of phages (Additional file 1: Fig. S6).

KpG phages were injected into zebrafish and toxicity due to phages were evaluated by assessing liver (alpha and beta naphthol) and brain (acetyl choline esterase) enzyme profiles of zebrafish. Phage administration did not cause any significant variation in liver enzyme profiles of zebrafish. But, discernible increase in brain acetyl choline esterase profiles were observed, which was not statistically significant $(\mathrm{P}=0.0529)$ (Additional file 1 : Fig. S7). Hematoxylin and eosin stained muscle and liver tissue from $\mathrm{KpG}$ phages treated group relative to phage untreated group also revealed that both muscle and liver tissue appeared normal and was similar to untreated control (Additional file 1: Fig. S8) implying that phage administration did not induce either morphological/biochemical aberrations in zebrafish.

Finally, to discern in vivo efficacy of phages, $10 \mu \mathrm{l}$ of MTCC 432 strain of $K$. pneumoniae corresponding to $\sim 1 \times 10^{5} \mathrm{CFU} / \mathrm{ml}$ was injected into muscle tissue of zebrafish. $2 \mathrm{~h}$ post infection, groups of 6 fishes were injected intramuscularly either with Streptomycin, KpG phages $\left(10^{8} \mathrm{PFU} / \mathrm{ml}\right)$ or a combination of KpG phages + Streptomycin and the ability of phages alone/in combination with antibiotics to curtail bacterial growth in infected muscle tissue was evaluated. Our results 
showed that relative to untreated control, streptomycin treatment caused $62.8 \%$ reduction in cell counts (CFU/ $\mathrm{ml}), \mathrm{KpG}$ phages treatment caused $77 \%$ reduction in cell counts and a combination of KpG phages + Streptomycin caused 98\% decline in cell counts in infected muscle tissue underscoring ability of phage antibiotic combination to significantly reduce bacterial bioburden. All treatments caused statistically significant decline in cell counts relative to untreated control (Fig. 6). This reiterates the fact that lytic phages from Ganges were not only effective in vitro against planktonic and biofilm mode of growth, it was also effective in vivo in curtailing bacterial growth in infected muscle tissue of zebrafish and hence has the potential to mitigate in vivo infection by $K$. pneumoniae in higher animal models.

\section{Discussion}

CRKP pose grave threat to public health especially in immune compromised patients and in neonates where mortality rate is very high (Investigators of the Delhi Neonatal Infection Study (DeNIS) collaboration 2016). CRKP classified as a critical priority pathogen by WHO, which severely limits therapeutic options available (Tumbarello et al. 2012). In this scenario, newer antibiotics are not the way out since bacteria will easily gain resistance to new antimicrobial agents due to evolutionary selection pressures. Although resistance modulators like

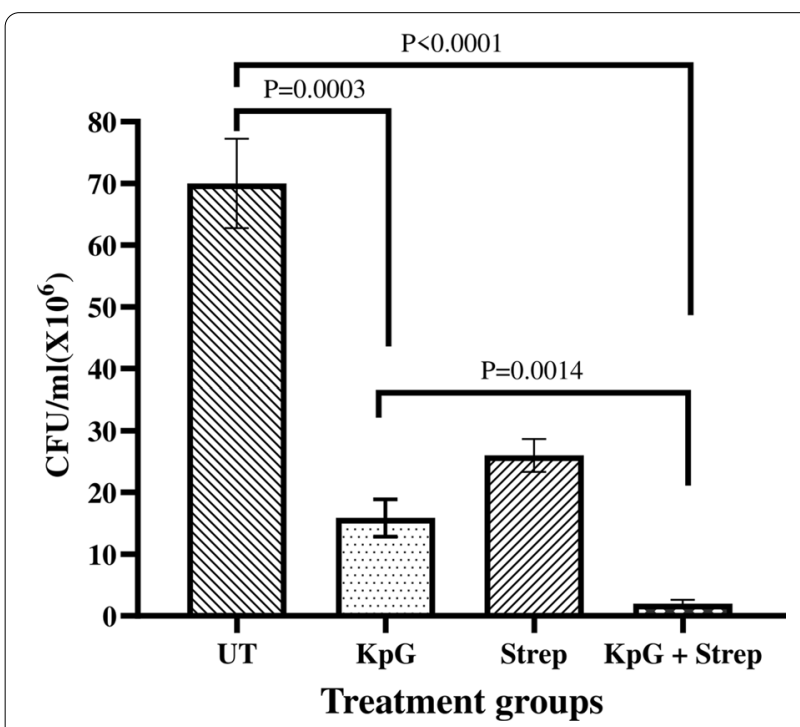

Fig. $6 \mathrm{KpG}$ phages reduced bacterial bioburden in infected fish. $10 \mu \mathrm{l}$ of K. pneumoniae $\left(10^{6} \mathrm{CFU} / \mathrm{ml}\right)$ were injected intramuscularly in zebrafish and $2 \mathrm{~h}$ post infection, $\mathrm{KpG}$ was administered intramuscularly. $24 \mathrm{~h}$ post infection, the muscle was dissected, serially diluted and plated on to LA plates. CFU/ml was calculated. Experiment was performed in triplicates and error bar represent the standard error of the mean betalactamase inhibitors, efflux pump inhibitors, quorum sensing inhibitors, cationic peptides are seen as a viable alternative, they were not as effective against MDR pathogens as expected. These bugs could circumvent these agents, for example, by producing betalactamases with metal co-factors that could not be inhibited by betalactamase inhibitors or by expressing redundant efflux transport proteins to extrude the antibiotics etc. Hence, to tackle AMR menace, biological control agents like lytic phages are considered as better alternatives (Aleshkin et al. 2016). The advantage of phage therapy is that it is highly specific and targeted hence it does not disturb commensal microbiota or lead to dysbiosis, given importance of commensal microbes in our health and wellbeing (Blander et al. 2017; Novince et al. 2017), whereas antibiotics could potentially harm commensal microbes resulting in dysbiosis.

Rivers like Ganges harbor a wide diversity of phages, which affords a rich source of targeted biological control agents against rising menace of drug resistant pathogens. The reason for thriving diversity of phages in Ganges river is that the Himalayan permafrost (source of Ganges river) upon melting, released abiotic phages that were trapped long time ago, contributing to unique diversity of bacteriophages (Khairnar 2016). This rich diversity of phages could be used as a viable source to curtail MDR pathogens in both planktonic and biofilm mode of growth. A previous report has also shown that a cocktail of phages isolated from Ganges river and sewage water against carbapenem and colistin resistant E. coli, K. pneumoniae and Enterobacter spp effectively reduced mixed bacterial load (Manohar et al. 2019).

In our study, the KpG from Ganges water was specific only for one clinical isolate obtained from urinary tract (MTCC 432 strain) and not towards other capsulated or uncapsulated strains of $K$. pneumoniae or E. coli or other bacterial species like $P$. aeruginosa, E. cloacae and A. baumannii. Similar to our observation, another report showed that four lytic phages, belonging to the family Podoviridae, infecting K. pneumoniae capsular type K22, were isolated from environmental samples. They possessed narrow infectivity only against a $K$. pneumoniae clinical isolates with K22 capsular type (Domingo-Calap et al. 2020). Moreover, the plaque morphology showed a halo around the lysis area, implying presence of depolymerase that lyses the capsule (Domingo-Calap et al. 2020). Interestingly, MTCC 432 strain produced capsule (Additional file 1: Fig. S1), as we observed a halo around the plaque (Additional file 1: Figs. S2, S3), it is likely that KpG phages possess capsular depolymerases (Oliveira et al. 2019). pH stability studies showed that KpG phages were unstable at lower $\mathrm{pH}$ and retained infectivity from $\mathrm{pH} 7.0$ to $\mathrm{pH}$ 11.0. Whereas, depolymerase activity as 
indicated by halo around the plaques was observed only until $\mathrm{pH}$ 9.0. Absence of halo at $\mathrm{pH} 11.0$ implies either lack of depolymerase expression or its inactivation. Since not even a faint zone was observed at $\mathrm{pH} 11.0$ we presumed that it is due to former cause rather than the latter, which remains to be confirmed by RT-PCR studies in future. Tropism of phages is a very well-established phenomenon. In an earlier study, a novel PhiKMV like virus infected only $K$. pneumoniae strains with $\mathrm{K} 1$ capsule but not the other capsular types. Capsule deleted K1 mutant strains could not be infected by this phage implying that capsule is essential for infection (Lin et al. 2014). On the other hand, broadly specific multi-host bacteriophage ФK64-1 produced nine different capsular depolymerases, which enabled this phage to infect 10 different $K$. pneumoniae possessing distinct capsular variants and mutants of these depolymerases failed to infect the corresponding strains (Pan et al. 2017). Capsular tropic phages are known in Klebsiella spp. Interestingly, a phage FC3-1 trophic to core polysaccharide and O antigen of LPS of $K$. pneumoniae was reported in an earlier study (Tomás and Jofre 1985).

KpG phages had a larger burst size 224 PFU/cell with a short latent period of $25 \mathrm{~min}$. A range of burst size has been reported with Klebsiella specific phages for example KPO1K2 displayed a burst size of $140 \mathrm{PFU} /$ infected cell (Verma et al. 2009). Another study had shown that Phage $\mathrm{Z}$ belonging to family Siphoviridae gave a burst size of 320 PFU/infected cell. Phage kpssk3 (Podoviridae) isolated from sewage, had a very short latent period of $10 \mathrm{~min}$ and a larger burst size of $200 \mathrm{pfu} / \mathrm{cell}$, which was able to lyse 25 clinical isolates of CRKP (Shi et al. 2020). Genome analysis of phage kpssk3 revealed that it did not possess any resistance genes, virulence factors and had depolymerase activity towards exopolysaccharide (Shi et al. 2020).

TEM imaging and sequence similarity to conserved T7 like phage primase/helicase and putative DNA primase of Podoviridae revealed that the Ganges phages belong to family Podoviridae. Most of the reported phages fall under family Siphoviridae, Podoviridae or Myoviridae (Kęsik-Szeloch et al. 2013). Significant biofilm inhibitory effect (Fig. 5a, 5b) coupled with the fact that MTCC strain produces capsule, imply that depolymerase of KpG phage might be effective against extracellular polymeric substances of biofilms, which remains to be explored in further studies. Significant proportion of dead cells by live/ dead imaging (Additional file 1: Fig. S7) imply that timing of addition of phages is important. Concomitant addition of both bacteria and phages to a localized solid surface causes lysis, whereas if bacteria were initially allowed to attach and initiate biofilm formation, subsequent addition of KpG phages after 1-2 h of bacterial interaction with glass surface did not cause significant bacterial lysis. Previous report has also shown that intranasal administration of phages $3 \mathrm{~h}$ prior infection or immediately after infection rescued mice from respiratory infection caused by $K$. pneumoniae strain whereas even $6 \mathrm{~h}$ delay in phage administration failed to rescue the mice highlighting importance of timing of phage administration (Chhibber et al. 2008). In an elegant study, to tackle mixed species biofilm formed by K. pneumoniae and Pseudomonas aeruginosa, wherein, Pseudomonas spp forms the bottom layer of the biofilm and is shielded by K. pneumoniae. The authors have used a combination of phages especially one that produces $K$. pneumoniae depolymerases, which disrupts the top layer and allows Pseudomonas aeruginosa specific phage to access inner biofilm layer. Klebsiella spp specific phages lacking depolymerase activity were unable to provide access for Pseudomonas spp. trophic phages. Lytic activity of phages was further enhanced in the presence of xylitol (Chhibber et al. 2015).

Among in vivo models, mice is commonly used to evaluate efficacy of bacteriophages in mitigating various infections ranging from burn wound, respiratory infections, sepsis and UTI (Capparelli et al. 2007; Malik and Chhibber 2009; Verma et al. 2009; Cao et al. 2015; Basu et al. 2015). To the best of our knowledge, zebrafish has not been evaluated as an animal model to evaluate bacteriophage therapy in infected fish. The advantages of zebrafish model is ease of availability and maintenance, optical clarity of embryo/larvae, short duration for study, a high degree of genetic homology with humans (Lieschke and Currie 2007). We have shown that injection of Klebsiella spp specific phages into adult zebrafish did not pose any toxicity as evidenced by liver and brain enzyme profiles and by histopathological analysis (Additional file 1: Figs. S5, S6). Earlier studies in mice have similarly shown lack of toxicity due to administration of phages and in addition, bioavailability of phages in various tissues were evident within $6 \mathrm{~h}$ post injection and half life of phages in mice was roughly $18 \mathrm{~h}$ (Verma et al. 2009). In our study, we observed a significant $77 \%$ decline in bacterial cell counts in infected muscle tissue relative to untreated control due to lytic activity of KpG phages. But combination of streptomycin + KpG phage was much more effective in reducing colony counts to $97 \%$ relative to untreated control. Previous study showed that in a mice full thickness wound model, when efficacy of phage therapy was compared with combination of gentamycin and silver nitrate to tackle infection by K. pneumoniae, it was observed that a single dose of phage as a topical application mitigated colonization of $K$. pneumoniae, whereas even multiple applications of silver nitrate and gentamycin failed to afford such protection (Kumari et al. 2011). In another study, same group showed that induced 
burn wounds in mice infected with MDR K. pneumoniae strain was successfully mitigated, with a significant decrease in bacterial load in blood and peritoneal lavage, when phages were administered either by subcutaneous or intraperitoneal route (Malik and Chhibber 2009). In the present study, high specificity of isolated KpG phage to a particular capsular strain of MTCC 432,synergy of phage with streptomycin in restricting regrowth in time kill assay, remarkable $\mathrm{pH}$ stability of phage from $\mathrm{pH} 7.0$ to 11.0. and significant biofilm inhibitory effect coupled with good in vivo effect in restricting bacterial growth in infected muscle tissue underscore the utility of $\mathrm{KpG}$ phages to curtail bacterial biofilms in vitro and restrict planktonic growth of Klebsiella spp. in vivo in zebrafish. Lack of toxicity to phages coupled with ease of performing the experiment indicates zebrafish can indeed serve as an initial in vivo model before evaluating efficiency of phages that could potentially mitigate MDR bacterial infections in higher animal models.

\section{Supplementary Information}

The online version contains supplementary material available at https://doi. org/10.1186/s13568-021-01181-0.

Additional file 1. Electronic supplementary information.

\section{Abbreviations}

CFU/ml: Colony forming units/ml; PFU/ml: Plaque forming units/ml; MOI: Multiplicity of infection; TEM: Transmission electron microscopy; PBS: Phosphate buffered saline; BHI: Brain heart infusion media; MIC: Minimum inhibitory concentration; MDR: Multidrug resistant; CRKP: Carbapenem resistant Klebsiella pneumoniae.

\section{Acknowledgements}

The financial support of SERB-DST, Government of India, under the EMR scheme (EMR/2016/001168) is earnestly acknowledged. Infrastructural support provided through DST-FIST funding (SR/FST/ETI-331/2013) is thankfully acknowledged. The authors would like to thank SASTRA for providing R\&M funds (R\&M/0039/SCBT-011/2017-2018)

\section{Authors' contributions}

NSS performed almost all the experiments and wrote the manuscript. ST carried out imaging studies. MMB participated in design of conserved primers, PCR amplification and sequence analysis. SN and GAK designed the study, performed data analysis and contributed to manuscript writing and editing.

\section{Funding}

The research work was funded by R\&M ((R\&M/0039/SCBT-011/2017-2018)

funds of SASTRA deemed University, Thanjavur, India.

\begin{abstract}
Availability of data and materials
Almost all data generated or analyzed during this study are included in this published article (and its Supplementary Information files). The raw data would be available upon request. KpG (phage lysate) can be obtained by contacting the corresponding author Dr. N. Saisubramanian (sai@scbt.sastra.edu).
\end{abstract}

\section{Ethics approval and consent to participate}

All applicable international, national, and/or institutional guidelines for the care and use of animals were followed.

\section{Consent for publication}

Not applicable.

\section{Competing interests}

The authors declare that they have no competing interests.

Received: 1 September 2020 Accepted: 11 January 2021

Published online: 15 February 2021

\section{References}

Abedon ST, Kuhl SJ, Blasdel BG, Kutter EM (2011) Phage treatment of human infections. Bacteriophage 1:66-85. https://doi.org/10.4161/bact.1.2.15845

Adams M (1959) Assay of phages by the agar layer method. In: Bacteriophages. Interscience Publishers, pp 450-451

Aleshkin A, Ershova O, Volozhantsev N, Svetoch E, Rubalsky E, Borzilov A, Aleshkin V, Afanasiev S, Bochkareva S (2016) Phagebiotics in treatment and prophylaxis of healthcareassociated infections. In: Bacteriophages: An Overview and Synthesis of a Re-Emerging Field. Nova Science Publishers, Inc., pp 105-122

Anand T, Virmani N, Kumar S, Mohanty AK, Pavulraj S, Bera BC, Vaid RK, Ahlawat U, Tripathi BN (2020) Phage therapy for treatment of virulent Klebsiella pneumoniae infection in a mouse model. J Glob Antimicrob Resist 21:34-41. https://doi.org/10.1016/j.jgar.2019.09.018

Andrews JM, Andrews JM (2001) Determination of minimum inhibitory concentrations. J Antimicrob Chemother 48(Suppl 1):5-16. https://doi. org/10.1093/jac/48.suppl_1.5

Basu S, Agarwal M, Bhartiya SK, Nath G, Shukla VK (2015) An in vivo wound model utilizing bacteriophage therapy of pseudomonas aeruginosa biofilms. Ostomy Wound Manag 61:16-23

Blander JM, Longman RS, lliev ID, Sonnenberg GF, Artis D (2017) Regulation of inflammation by microbiota interactions with the host. Nat Immunol 18:851-860

Bonilla N, Rojas MI, Cruz GNF, Hung SH, Rohwer F, Barr JJ (2016) Phage on tap-a quick and efficient protocol for the preparation of bacteriophage laboratory stocks. PeerJ 2016:e2261. https://doi.org/10.7717/peerj.2261

Burmeister AR, Fortier A, Roush C, Lessing AJ, Bender RG, Barahman R, Grant R, Chan BK, Turner PE (2020) Pleiotropy complicates a trade-off between phage resistance and antibiotic resistance. Proc Natl Acad Sci. https://doi. org/10.1073/pnas.1919888117

Cao F, Wang X, Wang L, Li Z, Che J, Wang L, Li X, Cao Z, Zhang J, Jin L, Xu Y (2015) Evaluation of the efficacy of a bacteriophage in the treatment of pneumonia induced by multidrug resistance Klebsiella pneumoniae in mice. Biomed Res Int 2015:752930. https://doi.org/10.1155/2015/752930

Capparelli R, Parlato M, Borriello G, Salvatore P, lannelli D (2007) Experimental phage therapy against Staphylococcus aureus in mice. Antimicrob Agents Chemother 51:2765-2773. https://doi.org/10.1128/AAC.01513-06

Chan BK, Sistrom M, Wertz JE, Kortright KE, Narayan D, Turner PE (2016) Phage selection restores antibiotic sensitivity in MDR Pseudomonas aeruginosa. Sci Rep 6:1-8. https://doi.org/10.1038/srep26717

Chhibber S, Bansal S, Kaur S (2015) Disrupting the mixed-species biofilm of klebsiella pneumoniae B5055 and pseudomonas aeruginosa PAO using bacteriophages alone or in combination with xylitol. Microbiol (United Kingdom) 161:1369-1377. https://doi.org/10.1099/mic.0.000104

Chhibber S, Kaur S, Kumari S (2008) Therapeutic potential of bacteriophage in treating Klebsiella pneumoniae B5055-mediated lobar pneumonia in mice. J Med Microbiol 57:1508-1513. https://doi.org/10.1099/ jmm.0.2008/002873-0

Christena LR, Raman T, Makala VH, Ulaganathan V, Subramaniapillai S (2016) Dithiazole thione derivative as competitive NorA efflux pump inhibitor to curtail Multi Drug Resistant clinical isolate of MRSA in a zebra fish infection model. Appl Microbiol Biotechnol. https://doi.org/10.1007/s0025 3-016-7759-2

Christensen GD, Simpson WA, Younger JJ, Baddour LM, Barrett FF, Melton DM, Beachey EH (1985) Adherence of coagulase-negative staphylococci to plastic tissue culture plates: a quantitative model for the adherence of staphylococci to medical devices. J Clin Microbiol 22:996-1006. https:// doi.org/10.1128/jcm.22.6.996-1006.1985

Domingo-Calap P, Beamud B, Vienne J, González-Candelas F, Sanjuán R (2020) Isolation of four lytic phages infecting Klebsiella pneumoniae K22 clinical isolates from Spain. Int J Mol Sci. https://doi.org/10.3390/ijms21020425

Dwivedi B, Schmieder R, Goldsmith DB, Edwards RA, Breitbart M (2012) PhiSiGns: An online tool to identify signature genes in phages and design 
PCR primers for examining phage diversity. BMC Bioinf 13:37. https://doi. org/10.1186/1471-2105-13-37

German GJ, Misra R (2001) The TolC protein of Escherichia coli serves as a cellsurface receptor for the newly characterized TLS bacteriophage. J Mo Biol 308:579-585. https://doi.org/10.1006/jmbi.2001.4578

Grillon A, Schramm F, Kleinberg M, Jehl F (2016) Comparative activity of ciprofloxacin, levofloxacin and moxifloxacin against Klebsiella pneumoniae, Pseudomonas aeruginosa and Stenotrophomonas maltophilia assessed by minimum inhibitory concentrations and time-kill studies. PLOS ONE 11:1-10. https://doi.org/10.1371/journal.pone.0156690

Holst Sørensen MC, van Alphen LB, Fodor C, Crowley SM, Christensen BB, Szymanski CM, Brøndsted L (2012) Phase variable expression of capsular polysaccharide modifications allows Campylobacter jejuni to avoid bacteriophage infection in chickens. Front Cell Infect Microbiol 2:11. https:// doi.org/10.3389/fcimb.2012.00011

Hoyles L, Murphy J, Neve H, Heller KJ, Turton JF, Mahony J, Sanderson JD, Hudspith B, Gibson GR, McCartney AL (2015) Sinderen D (2015) Klebsiella pneumoniae subsp pneumoniae-bacteriophage combination fromthe caecal effluent of a healthy woman. PeerJ. 12:4. https://doi.org/10.7717/ peerj.1061

Investigators of the Delhi Neonatal Infection Study (DeNIS) collaboration (2016) Characterisation and antimicrobial resistance of sepsis pathogens in neonates born in tertiary care centres in Delhi, India: a cohort study. Lancet Glob Heal 4:e752-e760. https://doi.org/10.1016/S2214 $-109 \times(16) 30148-6$

Jarvis WR, Munn VP, Highsmith AK, Culver DH, Hughes JM (1985) The epidemiology of nosocomial infections caused by Klebsiella pneumoniae. Infect Control 6:68-74

Kęsik-Szeloch A, Drulis-Kawa Z, Weber-Dąbrowska B, Kassner J, MajkowskaSkrobek G, Augustyniak D, Lusiak-Szelachowska M, Zaczek M, Górski A, Kropinski AM (2013) Characterising the biology of novel lytic bacteriophages infecting multidrug resistant Klebsiella pneumoniae. Virol J 10:100. https://doi.org/10.1186/1743-422X-10-100

Khairnar K (2016) Ganges: special at its origin. J Biol Res 23:16

Kumari S, Harjai K, Chhibber S (2011) Bacteriophage versus antimicrobial agents for the treatment of murine burn wound infection caused by Klebsiella pneumoniae B5055. J Med Microbiol 60:205-210. https://doi. org/10.1099/jmm.0.018580-0

Lieschke GJ, Currie PD (2007) Animal models of human disease: zebrafish swim into view. Nat Rev Genet 8:353-367

Lin T-L, Hsieh P-F, Huang Y-T, Lee W-C, Tsai Y-T, Su P-A, Pan Y-J, Hsu C-R, Wu M-C, Wang J-T (2014) Isolation of a bacteriophage and its depolymerase specific for K1 capsule of Klebsiella pneumoniae: implication in typing and treatment. J Infect Dis 210:1734-1744. https://doi.org/10.1093/infdi s/jiu332

Malik R, Chhibber S (2009) Protection with bacteriophage KØ1 against fatal Klebsiella pneumoniae-induced burn wound infection in mice. J Microbiol Immunol Infect 42:134-140

Manohar P, Tamhankar AJ, Lundborg CS, Nachimuthu R (2019) Therapeutic characterization and efficacy of bacteriophage cocktails infecting Escherichia coli, Klebsiella pneumoniae, and Enterobacter Species. Front Microbiol 10:574. https://doi.org/10.3389/fmicb.2019.00574

Merritt JH, Kadouri DE, O'Toole GA (2005) Growing and analyzing static biofilms current protocols in microbiology. Wiley, New York

Monferrer E, Domingo-Calap P (2019) Virus-host coevolution as a tool for controlling bacterial resistance to phage therapy. J Biotechnol Biomed 2:24. https://doi.org/10.26502/jbb.2642-91280013

Neely M, Pfeifer J, Caparon M (2002) Streptococcus-zebrafish model of bacterial pathogenesis. Infect Immun 70:3904-3914. https://doi.org/10.1128/ |Al.70.7.3904

Novince CM, Whittow CR, Aartun JD, Hathaway JD, Poulides N, Chavez MB, Steinkamp HM, Kirkwood KA, Huang E, Westwater C, Kirkwood KL (2017)
Commensal gut microbiota immunomodulatory actions in bone marrow and liver have catabolic effects on skeletal homeostasis in health. Sci Rep 7:5747. https://doi.org/10.1038/s41598-017-06126-x

Oliveira H, Mendes A, Fraga AG, Ferreira A, Pimenta Al, Mil-Homens D, Fialho AM, Pedrosa J, Azeredo J (2019) K2 capsule depolymerase is highly stable, is refractory to resistance, and protects larvae and mice from Acinetobacter baumannii sepsis. Appl Environ Microbiol. https://doi.org/10.1128/ AEM.00934-19

Pajunen M, Kiljunen S, Skurnik M (2000) Bacteriophage phiYeO3-12, specific for Yersinia enterocolitica serotype O:3, is related to coliphages T3 and T7. J Bacteriol 182:5114-5120. https://doi.org/10.1128/ jb.182.18.5114-5120.2000

Pan Y-J, Lin T-L, Chen C-C, Tsai Y-T, Cheng Y-H, Chen Y-Y, Hsieh P-F, Lin Y-T, Wang J-T (2017) Klebsiella Phage ФK64-1 encodes multiple depolymerases for multiple host capsular types. J Virol. https://doi.org/10.1128/jvi.02457-16

Phillips JB, Westerfield M (2014) Zebrafish models in translational research: tipping the scales toward advancements in human health. DMM Dis Model Mech 7:739-743. https://doi.org/10.1242/dmm.015545

Sasani MS, Fereshteh Eftekhar (2020) Potential of a bacteriophage isolated from wastewater in treatment of lobar pneumonia infection induced by Klebsiella pneumoniae in mice. https://doi.org/https://doi.org/10.1007/ s00284-020-02041-Z

Shi Y, Chen Y, Yang Z, Zhang Y, You B, Liu X, Chen P, Liu M, Zhang C, Luo X, Chen Y, Yuan Z, Chen J, Gong Y, Peng Y (2020) Characterization and genome sequencing of a novel T7-like lytic phage, kpssk3, infecting carbapenemresistant Klebsiella pneumoniae. Arch Virol 165:97-104. https://doi. org/10.1007/s00705-019-04447-y

Sulakvelidze A, Alavidze Z, Morris J (2001) Bacteriophage therapy. Antimicrob Agents Chemother 45:649-659

Summers WC (2012) The strange history of phage therapy. Bacteriophage 2:130-133. https://doi.org/10.4161/bact.20757

Tomás JM, Jofre JT (1985) Lipopolysaccharide-specific bacteriophage for Klebsiella pneumoniae C3. J Bacteriol 162:1276-1279

Tumbarello M, Viale P, Viscoli C, Trecarichi EM, Tumietto F, Marchese A, Spanu T, Ambretti S, Ginocchio F, Cristini F, Losito AR, Tedeschi S, Cauda R, Bassetti M (2012) Predictors of mortality in bloodstream infections caused by Klebsiella pneumoniae carbapenemase-producing K. pneumoniae: importance of combination therapy. Clin Infect Dis 55:943-950. https:// doi.org/10.1093/cid/cis588

Vading M, Nauclér P, Kalin M, Giske CG (2018) Invasive infection caused by Klebsiella pneumoniae is a disease affecting patients with high comorbidity and associated with high long-term mortality. PLoS ONE. https://doi. org/10.1371/journal.pone.0195258

Verma V, Harjai K, Chhibber S (2009) Characterization of a T7-like lytic bacteriophage of Klebsiella pneumoniae B5055: a potential therapeutic agent. Curr Microbiol 59:274-281. https://doi.org/10.1007/s00284-009-9430-y

Xie Y, Wahab L, Gill JJ (2018) Development and validation of a microtiter plate-based assay for determination of bacteriophage host range and virulence. Viruses. https://doi.org/10.3390/v10040189

Zhao J, Zhang Z, Tian C, Chen X, Hu L, Wei X, Li H, Lin W, Jiang A, Feng R, Yuan J, Yin Z, Zhao X (2019) Characterizing the biology of lytic bacteriophage

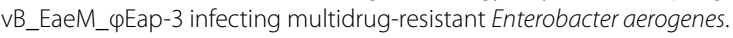
Front Microbiol 10:420. https://doi.org/10.3389/fmicb.2019.00420

World health Organisation (2017) Guidelines for the prevention and control of carbapenem-resistant Enterobacteriaceae, Acinetobacter baumannii and Pseudomonas aeruginosa in health care facilities. WHO, Geneva

\section{Publisher's Note}

Springer Nature remains neutral with regard to jurisdictional claims in published maps and institutional affiliations. 